\title{
Controlling the Growth Modes of Femtoliter Sessile Droplets Nucleating on Chemically Patterned Surfaces
}

\author{
Lei Bao, ${ }^{\dagger}$ Zenon Werbiuk, ${ }^{\dagger}$ Detlef Lohse, ${ }^{\ddagger}, \mathbb{I I}$ and Xuehua Zhang* ${ }^{* \dagger, 末}$ \\ ${ }^{\dagger}$ Soft Matter and Interfaces Group, School of Engineering, RMIT University, Melbourne, Victoria 3001, Australia \\ ${ }^{\ddagger}$ Physics of Fluids group, Department of Applied Physics and J. M. Burgers Centre for Fluid Dynamics, University of Twente, P.O. \\ Box 217, 7500 AE Enschede, The Netherlands \\ IIMax Planck Institute for Dynamics and Self-Organization, D-37077 Göttingen, Germany
}

ABSTRACT: Femtoliter droplet arrays on immersed substrates are essential elements in a broad range of advanced droplet-based technologies, such as light manipulation, sensing, and high throughput diagnosis. Solvent exchange is a bottom-up approach for producing those droplets from a pulse of oil oversaturation when a good solvent of the droplet liquid is displaced by a poor solvent. The position and arrangement of the droplets are regulated by chemical micropatterns on the substrate. Here we show experimentally and theoretically that the growth modes of droplets confined in planar micropatterns on the surface can be manipulated through the laminar flow of the solvent exchange. The control parameters are the area size of the micropatterns and the flow rate, and the observables are the contact angle and the final droplet volume. For a given pattern size, the Peclet number of the flow determines whether the growing droplets switch from an initial constant contact angle mode to a subsequent constant contact radius mode. Good agreement is achieved between the experimental results and our theoretical model that describes the dependence of the final droplet size on Pe.

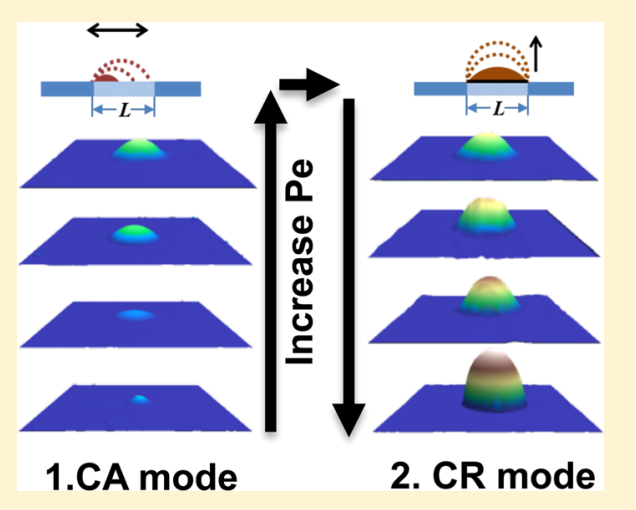

F emtoliter volume droplets immobilized on a substrate 1 provide the platform for miniaturization of physical, chemical, or biological processes. ${ }^{1-4}$ They are essential in microfludic systems, screening, crystallization, ${ }^{5-7}$ interfaceenhanced separation and purification, ${ }^{8,9}$ highly efficient heterogeneous catalysis, ${ }^{10}$ light manipulation, or high-resolution near-field imaging. ${ }^{11-13}$ Those droplet-based technologies rely on controlled generation of droplets with precise control over the droplet volume and position. Complementary to present top-down approaches of separating small volume droplets from a large bulk liquid, ${ }^{14,15}$ solvent exchange offers a simple bottom-up approach that is based on the nucleation and growth of nanodroplets on an immersed substrate. ${ }^{16-18}$ The position and arrangement of the droplets are well regulated by chemical micropatterns on the substrate fabricated by standard photolithgraphy. ${ }^{19}$

In the process of solvent exchange, an oil-saturated ethanol solution is displaced by oil-saturated water, which creates a pulse of oil oversaturation leading to the nucleation and growth of droplets. ${ }^{17}$ The solutions usually consist of three liquids (e.g., ethanol, water, and oil), among which one is miscible and the other two are immiscible liquids. The phase separation behavior of such ternary systems complicates the relation between the droplet size and the solution composition. Recently we have shown that the average volume of polydispersed droplets accumulated over a surface area increases with the flow rate of the solvent exchange, scaling with the Peclet number of the flow as $\propto \mathrm{Pe}^{3 / 4} \cdot{ }^{17,20}$ This mechanism promises an effective mean to tailor the droplet growth by flow conditions.

In this work, we show that the growth mode and final volume of highly ordered femtoliter oil droplets on chemical micropatterns are controlled by the flow conditions of the solvent exchange. We experimentally investigated the growth mode and final volume of droplets as a function of the Peclet number of the flow, and obtained good agreement with our theoretical prediction. To the best of our knowledge, this is the first quantitative study of the growth modes of femtoliter volume droplets in a laminar flow.

The solvent exchange was performed in a channel with welldefined flow geometries as sketched in Figure 1. The channel was first filled with oil-saturated 50\% ethanol aqueous solution, and then oil-saturated water was injected at a constant flow rate controlled by a syringe pump. Prepatterned substrates were placed side-by-side on the bottom of the horizontal channel. The micropatterns on the substrate were highly regular arrays of hydrophobic circular microdomains prepared by standard photolithography and silanization. ${ }^{19}$ The diameter of the microdomains was $3 \mu \mathrm{m}, 5 \mu \mathrm{m}$, and $10 \mu \mathrm{m}$, respectively, with a rim-to-rim distance twice the domain diameter. After the completion of the solvent exchange, droplet arrays formed on 
(a)

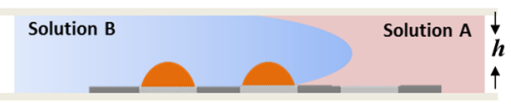

(b)

(c)
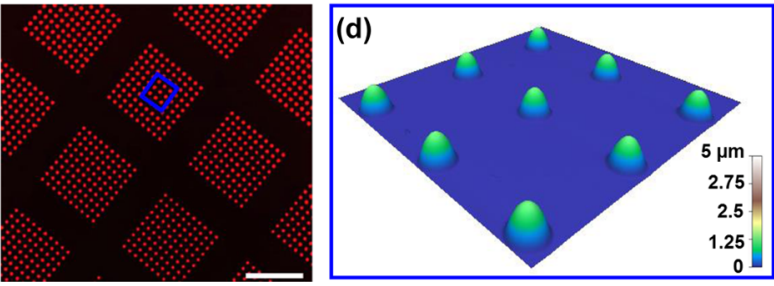

Figure 1. Formation and characterization of droplets on hydrophobic patterns. (a) Sketch of the solvent exchange process. (b) Sketch and notation of a surface droplet. $L$ is the lateral diameter, $\theta$ the contact angle, $H$, the maximum height of the droplet, and $R$ the radius of curvature. (c) Confocal image of the arrays of droplets dyed with Rhodamine 6G. The length of the scale bar is $15 \mu \mathrm{m}$. (d) Representative AFM image of droplets on $5 \mu \mathrm{m}$ patterns. The scan range is $50 \times 50 \mu \mathrm{m}^{2}$.

the substate, following the location of the hydrophobic patterns as shown in the confocal image in Figure 1c. Those droplet arrays were polymerized and characterized by atomic force microscopy as shown in a representative image in Figure $1 \mathrm{~d}$.

AFM images of the droplet and their cross sectional profiles are shown in Figure 2 as the Peclet number (Pe) of the flow increased from $\sim 10$ to 1200 . More images are provided in the Supporting Information. A general feature is that the droplets are larger at larger Pe number. On the patterns with a diameter
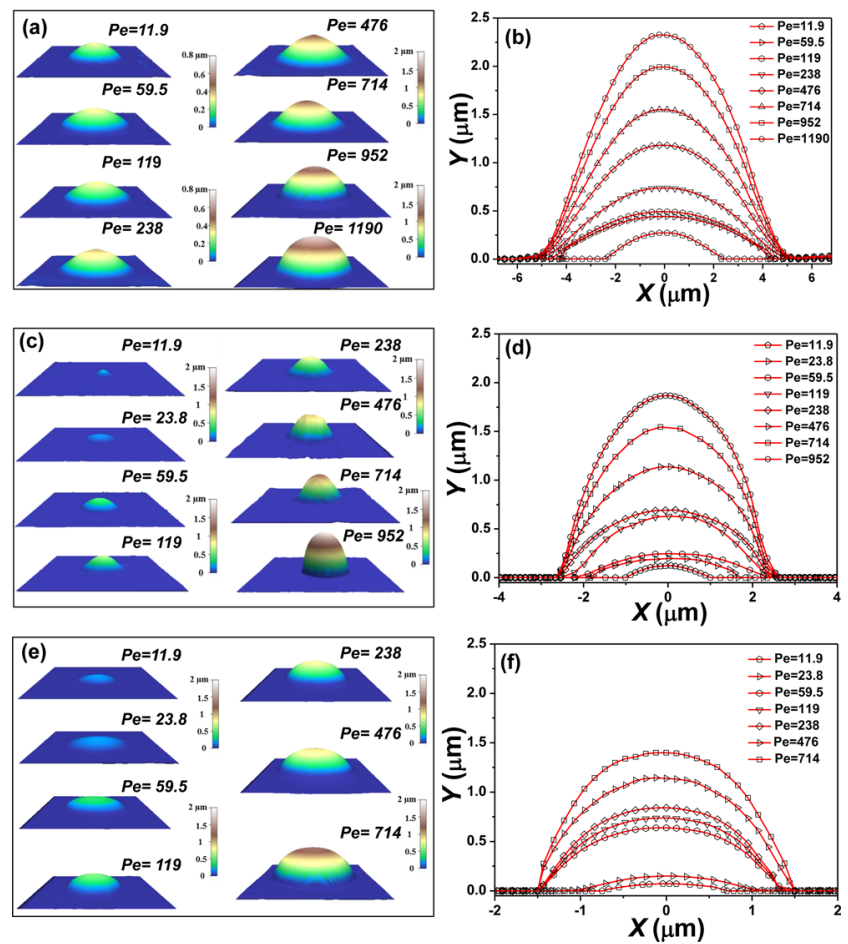

Figure 2. AFM images and cross-sectional profiles of droplets produced by the solvent exchange at different $\mathrm{Pe}$ on $(\mathrm{a}, \mathrm{b}) 10 \mu \mathrm{m}$ pattern, (c,d) $5 \mu \mathrm{m}$ pattern and (e,f) $3 \mu \mathrm{m}$ pattern. The scan range is $15 \times 15 \mu \mathrm{m}^{2}(\mathrm{a}, \mathrm{c})$ and $5 \times 5 \mu \mathrm{m}^{2}(\mathrm{e})$. All the profiles are aligned through the droplet center. $\mathrm{Pe}=U h / D$ is the Peclet number of the flow, where $U$ is the flow velocity, $h$ is channel height, and $D$ is diffusion coefficient. of $10 \mu \mathrm{m}$, the base diameter of the droplet is less than the pattern diameter when $\mathrm{Pe}=12$ or 23 . The droplet reached the rim of the pattern at $\mathrm{Pe}=60$ and remained the same base diameter at $\mathrm{Pe}$ up to 714, while the height increased from 50 to $1500 \mathrm{~nm}$. On the patterns of $5 \mu \mathrm{m}$ and $3 \mu \mathrm{m}$ in diameter, the droplet did not reach the rim of the domain until Pe was more than 120 and 200, respectively.

We plot the lateral diameter, height, volume, and contact angle of droplets as a function of $\mathrm{Pe}$ in Figure 3. The plots
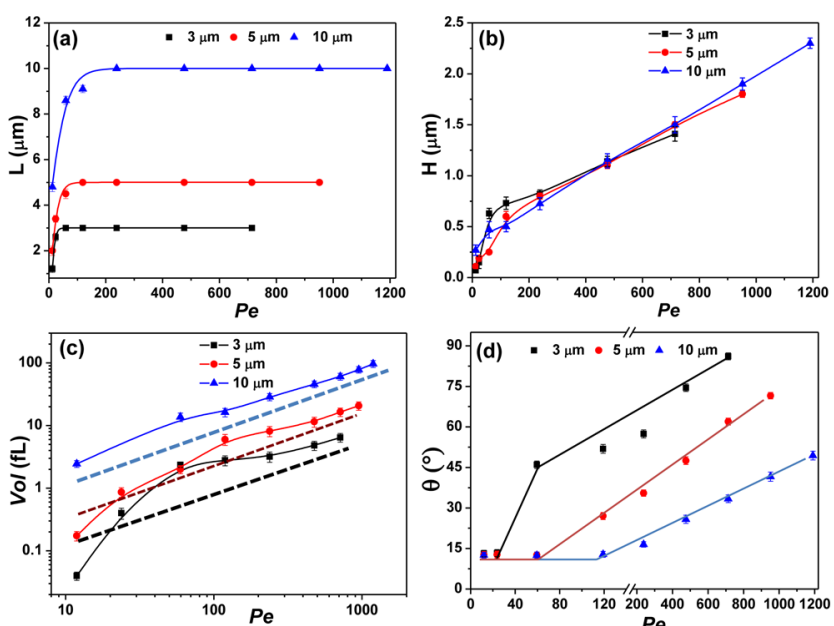

Figure 3. Morphological characteristics of droplets as a function of Pe. The plots of (a) lateral diameter, (b) height, (c) volume, and (e) contact angle of droplets on the patterns of $3 \mu \mathrm{m}, 5 \mu \mathrm{m}$, and $10 \mu \mathrm{m}$ as a function of Pe. The three dotted lines in (c) is the fitting from $\mathrm{Pe}^{3 / 4}$ as predicted for droplet growth in the CA mode. ${ }^{17}$

clearly show that there are two growth modes of the droplets: the constant contact angle mode (CA mode) and the constant contact radius (or area) mode (CR mode). In the CA mode, the lateral diameter and height of droplets increase rapidly with an increase in $\mathrm{Pe}$, resulting in a fast increase in the droplet volume. The contact angle of the droplets remain $\sim 13^{\circ}$ on all three sizes of patterns in this mode until $\mathrm{Pe}$ is above 24,60 , and 120 on the pattern of 3,5 , and $10 \mu \mathrm{m}$, respectively. As the lateral diameter of droplets reaches their respective pattern size, the CR mode kicks in, and the droplet diameter remains the same regardless of Pe. From then on, the height and contact angle of droplets gradually increase with an increase in Pe. The height of droplets on three patterns all reaches $\sim 1.5 \mu \mathrm{m}$ at $\mathrm{Pe}$ of 714 , while the contact angle is $33^{\circ}, 61^{\circ}$, and $86^{\circ}$ on 10,5 , and $3 \mu \mathrm{m}$ patterns, respectively.

The increase rate in the droplet volume in CR mode remains almost the same on the $10 \mu \mathrm{m}$ patterns, but slows down on the $5 \mu \mathrm{m}$ and on $3 \mu \mathrm{m}$ patterns. The droplet volume scaling with $\propto$ $\mathrm{Pe}^{3 / 4}$ well applies to all the patterns of $5 \mu \mathrm{m}$ and $10 \mu \mathrm{m}$, but deviates on $3 \mu \mathrm{m}$ patterns. The theoretical derivation of the $\mathrm{Pe}^{3 / 4}$ scaling law was based on the undisturbed growth of droplets in the CA mode. ${ }^{17}$ The deviation of the droplet volume from $\mathrm{Pe}^{3 / 4}$ scaling clearly suggests that the effect of the CA mode in early growth stage is more pronounced on the final volume of droplets on smaller patterns.

The two modes in the droplet growth are also visualized in the in situ video in the Supporting Information and the time course snapshots provided in Figure 4. Initially the solution was clear, and the substrate was smooth. As the moving front of the second solution passing by, multiple oil droplets appeared in a 

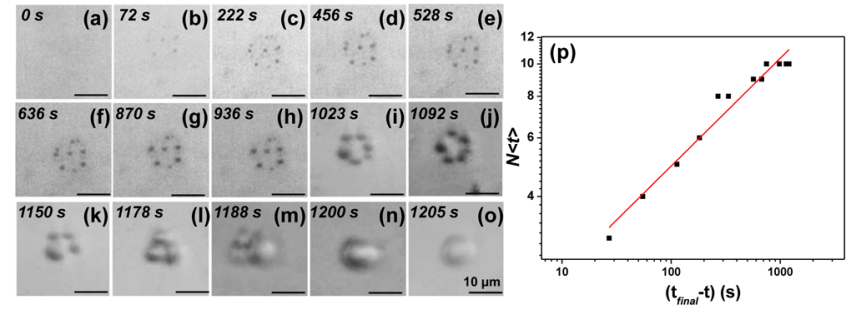

Figure 4. $(\mathrm{a}-\mathrm{o})$ The time evolution of droplets nucleation and growth on a circular hydrophobic miscrodomain with diameter of $10 \mu \mathrm{m}$. (p) Logrithmic plot of the droplet number as a function of time $(t)$.

circle at the rim of those circular micropatterns (Figure 3). The circles of droplets clearly suggest that the preferential sites for the droplet nucleation are the edge between the hydrophobic microdomains and the hydrophilic surrounding area, which is attributed to the relatively low energy barrier therein for the droplet nucleation. The number of droplets was found to be constant for a period of the solvent exchange, while all the droplets in the same pattern become bigger with time (Figure $4 c-h)$. Then the number of droplets decreases from 9 , to 7 , to 6 , and so on until only one droplet for a given domain. During the coalescence of neighboring droplets, two smaller seed droplets merged to one "new" droplet (Figure 4i-n). During this transition, the volume of the droplets gained and the shape of merged droplets become irregular. Eventually, one big droplet with lateral diameter of $10 \mu \mathrm{m}$ was achieved (Figure $40)$. This process is reminiscent of the growing droplets around the rim of a microcap. ${ }^{21}$

We plotted the time evolution of the droplet number $N(t)$ on the hydrophobic microdomain in Figure 4p. We find $N(t) \sim$ $t^{0.32}$, which is consistent with the mechanism of Ostwald ripening suggested by Lifshitz and Slyozov. ${ }^{22}$ Clearly, the droplet growth on the pattern surface follows the constant contact angle mode first and then the constant contact area mode. Those growth modes are also applicable for the droplets nucleating and growing on the circular hydrophobic microdomain with diameter of $5 \mu \mathrm{m}$ (Figure $2 \mathrm{c}, \mathrm{d}$ ) and $3 \mu \mathrm{m}$ (Figure $2 \mathrm{e}, \mathrm{f})$.

The droplet nucleation starts with the formation of many initial nanodroplets at the boundary of the circular hydrophobic domain shown in Figure 4. These oil nanodroplets will then grow and merge up to the time that the microdomain is fully covered. Assuming that in this early phase the nanodroplets grow in the constant contact angle mode (CA-mode) as sketched in Figure 5a, we can then adopt the theoretical
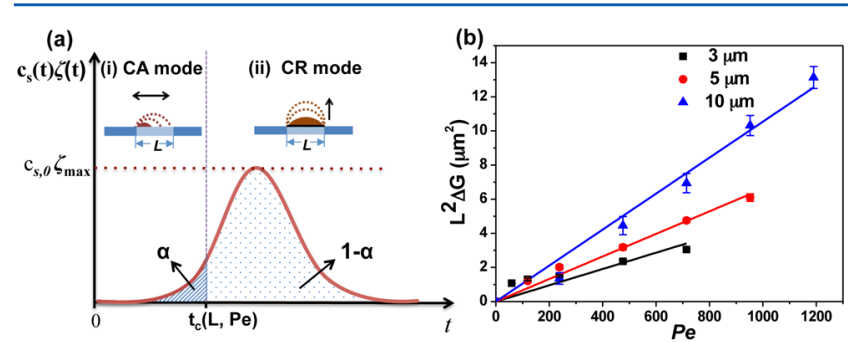

Figure 5. (a) Sketch of the two different growth modes and oversaturation level $c_{s}(t) \zeta(t)$ as a function of time. (b) Plots of $L^{2} \Delta G$ as a function of Pe. The slope (being equal to $L_{\tau}^{2}$ ) for the case $L=3 \mu \mathrm{m}$ is $0.48 \times 10^{-14} \mathrm{~m}^{2}$, that for $5 \mu \mathrm{m}$ is $0.66 \times 10^{-14} \mathrm{~m}^{2}$, and that for 10 $\mu \mathrm{m}$ is $1.1 \times 10^{-14} \mathrm{~m}^{2}$. framework of our earlier work ${ }^{17}$ and write for the growth dynamics of an individual droplet

$$
R \dot{R} \sim \frac{D}{\rho_{\mathrm{d}}} \sqrt{\operatorname{Pe}} c_{\mathrm{s}}(t) \zeta(t)
$$

where $R(t)$ is the radius of curvature of the oil droplet, $\rho_{\mathrm{d}}$ its density, $D$ the diffusion constant of oil in the surrounding liquid, and $c_{\mathrm{s}}(t)$ the time dependent saturation concentration during the solvent exchange process. Here $\mathrm{Pe}=U L / D$ is the Peclet number of the flow. The oversaturation pulse $c_{\mathrm{s}}(t) \zeta(t)=$ $c_{\infty}(t)-c_{s}(t)$ has Gaussian shape and can be described by a maximum concentration $c_{s, 0} \zeta_{\max }$ and a pulse width $\tau$.

Here in first approximation, we neglect that the local concentration field is affected by the competitive growth of the neighboring droplets. ${ }^{21}$ The growth then continues to obey eq 1 up to time $t_{1}$ and radius of curvature $R_{1}$ when the droplet merges with a neighboring droplet, which is assumed to have evolved through the same process, and thus has the same size $R_{1}$. To obtain the droplet size, we integrate eq 1 from 0 to $R_{1}$ and from 0 to $t=t_{1}$

$$
R_{1}^{2} \sim \frac{D c_{\mathrm{s}, 0}}{\rho_{\mathrm{d}}} \zeta_{\max } \tau P e^{1 / 2} \operatorname{erf}\left(t_{1}\right)
$$

The volume $V_{1}^{\prime} \sim R_{1}^{\prime 3}$ of the merged droplet with radius of curvature $R_{1}^{\prime}$ is twice the volume $V \sim R_{1}^{3}$ of the original droplet, i.e., $R_{1}^{\prime}=2^{1 / 3} R_{1}$. The merged droplet continues to grow due to the oversaturation up to time $t_{2}$ when it has size $R_{2}$ and again merges, leading to a droplet of size $R_{2}^{\prime}=2^{1 / 3} R_{2}$. We obtain $R_{2}$ by integrating eq 1 from $t_{1}$ to $t_{2}$ respective $R_{1}^{\prime}$ to $R_{2}$, giving

$$
R_{2}^{2}-R_{1}^{\prime 2} \sim \frac{D c_{\mathrm{s}, 0}}{\rho_{\mathrm{d}}} \zeta_{\max } \tau \operatorname{Pe}^{1 / 2}\left(\operatorname{erf}\left(t_{2}\right)-\operatorname{erf}\left(t_{1}\right)\right)
$$

The process repeats, after $i$ 'cascading' steps leading to

$$
R_{i}^{2}-2^{2 / 3} R_{i-1}^{2} \sim \frac{D c_{\mathrm{s}, 0}}{\rho_{\mathrm{d}}} \zeta_{\max } \tau \operatorname{Pe}^{1 / 2}\left(\operatorname{erf}\left(t_{i}\right)-\operatorname{erf}\left(t_{i-1}\right)\right)
$$

The CA growth process ends after $N_{c}$ cascading steps at the crossover time $t_{c}$ when the droplet contact diameter has reached the diameter $L$ of the hydrophobic domain size. This crossover time $t_{c}$ in general will depend on $\mathrm{L}$ and $\mathrm{Pe}$. eq 3 can be summed up, where on the right-hand side we make use of the telescope sum character,

$$
\sum_{i=1}^{N_{c}}\left(R_{i}^{2}-2^{2 / 3} R_{i-1}^{2}\right) \sim \frac{D c_{s, 0}}{\rho_{\text {oil }}} \zeta_{\max } \tau P e^{1 / 2} \operatorname{erf}\left(t_{c}(L, P e)\right)
$$

The left-hand side of this equation is $\sim L^{2}$, so our final result for the growth phase in the CA mode is

$$
L^{2} \sim \frac{D c_{\mathrm{s}, 0}}{\rho_{\mathrm{d}}} \zeta_{\max } \tau \mathrm{Pe}^{1 / 2} \operatorname{erf}\left(t_{\mathrm{c}}(L, \mathrm{Pe})\right)
$$

$\alpha(L, \mathrm{Pe})=\operatorname{erf}\left(t_{\mathrm{c}}(L, \mathrm{Pe})\right)$ can be seen as fraction of the oil oversaturation pulse, which is used for growth in the CA-mode. Obviously, $0 \leq \alpha(L, \mathrm{Pe}) \leq 1$.

From time $t_{\mathrm{c}}$ on the droplet will grow in the constant contact radius mode (CR-mode), starting with the contact angle $\theta_{\mathrm{c}}$ to the final contact angle $\theta_{\mathrm{f}}$. For the droplet growth purely governed by diffusion, i.e., in the absence of any external flow, the evolution of the contact angle $\theta$ would be given by ${ }^{18,23}$ 


$$
\frac{\mathrm{d} \theta}{\mathrm{d} t}=\frac{4 D}{\rho_{\mathrm{d}} L^{2}}(1+\cos \theta)^{2} f(\theta) c_{\mathrm{s}}(t) \zeta(t)
$$

with $^{23}$

$$
f(\theta)=\frac{\sin \theta}{1+\cos \theta}+4 \int_{0}^{\infty} \frac{1+\cosh 2 \theta \xi}{\sinh 2 \pi \xi} \tanh [(\pi-\theta) \xi] \mathrm{d} \xi
$$

How does the situation change once we have an enhanced flow rate over the growing droplets, as in our experimental situation and as assumed already for the growth in the CRmode? Again we can make some qualitative statements, similarly as in ref 17 . We expect that the flow rate enhances the growth process as the concentration boundary layer (BL) around the droplet will become thinner thanks to the flow, with the length scale $L$ replaced by $L / \sqrt{\mathrm{Pe}}$, according to the Prandtl-Blasius-Pohlhausen result $\lambda \sim L / \sqrt{\mathrm{Pe}}$ for the width $\lambda$ of the concentration boundary layer. ${ }^{24,25}$ For large enough flow $\mathrm{Pe}>1$ the BL becomes thinner, and the concentration gradient larger than for the purely diffusive case, leading to enhanced growth thus described by

$$
\frac{\mathrm{d} \theta}{\mathrm{d} t} \sim \frac{4 D \mathrm{Pe}}{\rho_{\mathrm{d}} L^{2}}(1+\cos \theta)^{2} f(\theta) c_{\mathrm{s}}(t) \zeta(t)
$$

We integrate eq 8 from $t_{c}(L, \mathrm{Pe})$ to $\infty$ or respectively the contact angle $\theta_{\mathrm{c}}$ at the beginning of the CR mode to the final contact angle $\theta_{\mathrm{f}}$,

$$
\begin{aligned}
& G\left(\theta_{\mathrm{f}}\right)-G\left(\theta_{\mathrm{c}}\right):=\int_{\theta_{\mathrm{c}}}^{\theta_{\mathrm{f}}} \frac{\mathrm{d} \theta}{(1+\cos \theta)^{2} f(\theta)} \\
& \sim \frac{D \mathrm{Pe}}{\rho_{\mathrm{d}} L^{2}} \int_{t_{\mathrm{c}}(L, \mathrm{Pe})}^{\infty} c_{\mathrm{s}}(t) \zeta(t) \mathrm{d} t=\frac{D c_{\mathrm{s}, 0}}{\rho_{\mathrm{d}} L^{2}} \zeta_{\max } \tau \operatorname{Pe}(1-\alpha(L, \mathrm{Pe}))
\end{aligned}
$$

where as above we have assumed a Gaussian shape of the oversaturation pulse $c_{s}(t) \zeta(t)$. The first equal sign in eq 9 is the defining equation for the function $G(\theta)$ and $1-\alpha(\mathrm{L}, \mathrm{Pe})$ is obviously the fraction of time the droplet is growing in the $\mathrm{CR}$ mode. With this notation we can compactly write

$$
\theta_{\mathrm{f}}=G^{-1}\left(G\left(\theta_{\mathrm{c}}\right)+\text { const } \frac{D}{L^{2}} \frac{c_{\mathrm{s}, 0}}{\rho_{\mathrm{d}}} \zeta_{\max } \tau \operatorname{Pe}(1-\alpha(L, \mathrm{Pe}))\right)
$$

The final droplet height $H_{\mathrm{f}}$ and the final droplet volume $V_{\mathrm{f}}$ follow as

$$
H_{\mathrm{f}}=\frac{1-\cos \theta_{\mathrm{f}}}{2 \sin \theta_{\mathrm{f}}} L
$$

and

$$
V_{\mathrm{f}}=\frac{\pi}{24} H_{\mathrm{f}}\left(3 L^{2}+4 H_{\mathrm{f}}^{2}\right)
$$

respectively.

Our experimental results are summarized in Table 1. The crossover angle $\theta_{\mathrm{c}}$ is found to be the contact angle on the

\begin{tabular}{|c|c|c|c|c|c|c|}
\hline \multirow{2}{*}{$\frac{\mathrm{n} / \mathrm{a}}{\mathrm{Pe}}$} & \multicolumn{2}{|c|}{$\mathrm{L}=3 \mu \mathrm{m}$} & \multicolumn{2}{|c|}{$\mathrm{L}=5 \mu \mathrm{m}$} & \multicolumn{2}{|c|}{$\mathrm{L}=10 \mu \mathrm{m}$} \\
\hline & $\theta_{\mathrm{f}}$ & $\Delta G$ & $\theta_{\mathrm{f}}$ & $\Delta G$ & $\theta_{\mathrm{f}}$ & $\Delta G$ \\
\hline 59.5 & 46 & 0.119 & & $\mathrm{n} / \mathrm{a}$ & $\mathrm{n} / \mathrm{a}$ & $\mathrm{n} / \mathrm{a}$ \\
\hline 119 & 51.9 & 0.144 & 27 & 0.048 & $\mathrm{n} / \mathrm{a}$ & $\mathrm{n} / \mathrm{a}$ \\
\hline 238 & 57.4 & 0.166 & 35.5 & 0.080 & 16.5 & 0.014 \\
\hline 476 & 74.5 & 0.262 & 47.5 & 0.127 & 25.8 & 0.045 \\
\hline 714 & 86.1 & 0.339 & 61.95 & 0.190 & 33.4 & 0.069 \\
\hline 952 & $\mathrm{n} / \mathrm{a}$ & $\mathrm{n} / \mathrm{a}$ & 71.54 & 0.244 & 41.6 & 0.103 \\
\hline 1190 & $\mathrm{n} / \mathrm{a}$ & $\mathrm{n} / \mathrm{a}$ & $\mathrm{n} / \mathrm{a}$ & $\mathrm{n} / \mathrm{a}$ & 49.4 & 0.131 \\
\hline
\end{tabular}
hydrophobic domain, namely $\theta_{c}=13^{\circ}$ for all different cases characterized through their $\mathrm{L}$ and $\mathrm{Pe}$. As for all these cases, the factor $D c_{s, 0} \zeta_{\max } \tau / \rho_{d}$ is also the same, thus we can rewrite eq 9 as

$$
\Delta G:=G\left(\theta_{\mathrm{f}}\right)-G\left(\theta_{\mathrm{c}}\right)=\frac{L_{\tau}^{2}}{L^{2}} \operatorname{Pe}(1-\alpha(L, \mathrm{Pe}))
$$

Table 1. Summary of the Experimental Results for $\boldsymbol{\theta}_{\mathrm{f}}$, and $G$ $\left(\theta_{\mathrm{f}}\right)-\boldsymbol{G}\left(\boldsymbol{\theta}_{\mathrm{c}}\right)=: \Delta G(\theta)^{a}$

with a length scale $L_{\tau} \sim \sqrt{D c_{\mathrm{s}, 0} \zeta_{\max } \tau / \rho_{\mathrm{d}}}$, in which we have absorbed the unknown dimensionless prefactor of eq 9 and which characterizes the oversaturation pulse. The physical interpretation of $L_{\tau}$ is the one as diffusive length scale belonging to the time scale $\tau$ of the oversaturation pulse, for the oil of solubility $c_{\mathrm{s}, 0}$ and density $\rho_{\mathrm{d}}$, suggested by Epstein and Plesset. ${ }^{26}$

Equation 13 suggests to calculate $L^{2} \Delta G$ for our experimentally measured data of Table 1 and plot it as a function of $\mathrm{Pe}$ in Figure 5. Indeed, the data follow a linear trend with $\mathrm{Pe}$ as suggested by eq 13 , reflecting that the Pe-dependence of 1 $\alpha(\mathrm{L}, \mathrm{Pe})$ is only weak. The slope of these curves equals $L_{\tau}^{2}$, which implies $L \tau=70 \mathrm{~nm}$ for the $\mathrm{L}=3 \mu \mathrm{m}$ case, $L \tau=80 \mathrm{~nm}$ for the $\mathrm{L}=5 \mu \mathrm{m}$ case, and $L_{\tau}=105 \mathrm{~nm}$ for the $\mathrm{L}=10 \mu \mathrm{m}$ case, quite similar to each other, as one would expect from the theory.

In summary, we demonstrate that the growth modes and size of femtoliter droplets can be controlled by simple flow conditions of the solvent exchange. The experimental results clearly show that the Pe number of the flow determined whether the growth of droplets confined in a certain sized micropatterns switch from an initial constant contact angle mode to a sequential constant contact area mode. We have theoretically analyzed the two growth modes of femtoliter droplets in the confined area that predicts the dependence of the contact angle of droplets on Pe number. The experimental results and theoretical prediction are in good agreement. Our work provides a novel approach based on the flow conditions for tailoring the size and morphology of surface nanodroplets in highly ordered array.

*E-mail: xuehua.zhang@rmit.edu.au.

\section{Notes}

The authors declare no competing financial interest. 


\section{ACKNOWLEDGMENTS}

L.B. acknowledges the support from a VC Postdoctoral Fellowship from RMIT University, X.H.Z. from the Australian Research Council (FT120100473, DP140100805) and NWO MCEC program, and D.L. from an ERC-Advanced Grant and the MCEC program.

\section{REFERENCES}

(1) Vaitheeswaran, S.; Thirumalai, D. J. Am. Chem. Soc. 2006, 128, 13490-13496. PMID: 17031962.

(2) Méndez-Vilas, A.; Jódar-Reyes, A. B.; González-Martín, M. L. Small 2009, 5, 1366-1390.

(3) Chiu, D. T.; Lorenz, R. M. Acc. Chem. Res. 2009, 42, 649-658. PMID: 19260732.

(4) Xu, H.; Zhang, X. Adv. Colloid Interface Sci. 2015, 224, 17-32.

(5) Stephens, C. J.; Kim, Y.-Y.; Evans, S. D.; Meldrum, F. C.; Christenson, H. K. J. Am. Chem. Soc. 2011, 133, 5210-5213. PMID: 21425847.

(6) Shemesh, J.; Ben Arye, T.; Avesar, J.; Kang, J. H.; Fine, A.; Super, M.; Meller, A.; Ingber, D. E.; Levenberg, S. Proc. Natl. Acad. Sci. U. S. A. 2014, 111, 11293-11298.

(7) Hu, S.-W.; Xu, B.-Y.; Ye, W.-k.; Xia, X.-H.; Chen, H.-Y.; Xu, J.-J. ACS Appl. Mater. Interfaces 2015, 7, 935-940. PMID: 25525675.

(8) Li, H.; Yang, Q.; Li, G.; Li, M.; Wang, S.; Song, Y. ACS Appl. Mater. Interfaces 2015, 7, 9060-9065. PMID: 25761507.

(9) Shi, X.; Chen, C.-H.; Gao, W.; Chao, S.-h.; Meldrum, D. R. Lab Chip 2015, 15, 1059-1065.

(10) Kim, B.-K.; Kim, J.; Bard, A. J. J. Am. Chem. Soc. 2015, 137, 2343-2349. PMID: 25616104.

(11) Lee, J. Y.; Hong, B. H.; Kim, W. Y.; Min, S. K.; Kim, Y.; Jouravlev, M. V.; Bose, R.; Kim, K. S.; Hwang, I.-C.; Kaufman, L. J.; Wong, C. W.; Kim, P.; Kim, K. S. Nature 2009, 460, 498-501.

(12) Wang, Z.; Guo, W.; Li, L.; Luk'yanchuk, B.; Khan, A.; Liu, Z.; Chen, Z.; Hong, M. Nat. Commun. 2011, 2, 218.

(13) Chen, Y.; Elshobaki, M.; Ye, Z.; Park, J.-M.; Noack, M. A.; Ho, K.-M.; Chaudhary, S. Phys. Chem. Chem. Phys. 2013, 15, 4297-4302.

(14) Kuang, M.; Wang, L.; Song, Y. Adv. Mater. 2014, 26, 69836983.

(15) Sun, J.; Bao, B.; He, M.; Zhou, H.; Song, Y. ACS Appl. Mater. Interfaces 2015, 7, 28086-28099. PMID: 26642390.

(16) Zhang, X. H.; Ducker, W. Langmuir 2008, 24, 110-115.

(17) Zhang, X.; Lu, Z.; Tan, H.; Bao, L.; He, Y.; Sun, C.; Lohse, D. Proc. Natl. Acad. Sci. U. S. A. 2015, 112, 9253-9257.

(18) Lohse, D.; Zhang, X. Rev. Mod. Phys. 2015, 87, 981-1035.

(19) Bao, L.; Rezk, A. R.; Yeo, L. Y.; Zhang, X. Small 2015, 11, $4850-4855$

(20) Yu, H.; Lu, Z.; Lohse, D.; Zhang, X. Langmuir 2015, 31, 1262812634. PMID: 26514099.

(21) Peng, S.; Lohse, D.; Zhang, X. ACS Nano 2015, 9, 1191611923.

(22) Lifshitz, I. M.; Slyozov, V. V. J. Phys. Chem. Solids 1961, 19, 3550.

(23) Popov, Y. O. Phys. Rev. E 2005, 71, 036313.

(24) Schlichting, H. Boundary Layer Theory, 7th ed.; McGraw Hill: New York, 1979.

(25) Grossmann, S.; Lohse, D. Phys. Fluids 2004, 16, 4462-4472.

(26) Epstein, P. S.; Plesset, M. S. J. Chem. Phys. 1950, 18, 1505. 